\title{
Genetically Determined Height and Risk of Non-hodgkin Lymphoma
}

\begin{abstract}
Amy Moore ${ }^{1}$, Eleanor Kane ${ }^{2}$, Zhaoming Wang ${ }^{3,4}$, Orestis A. Panagiotou ${ }^{5,6}$, Lauren R. Teras ${ }^{7}$, Alain Monnereau ${ }^{8,9,10}$, Nicole Wong Doo ${ }^{11}$, Mitchell J. Machiela ${ }^{1}$, Christine F. Skibola ${ }^{12}$, Susan L. Slager ${ }^{13}$, Gilles Salles ${ }^{14,15,16}$, Nicola J. Camp ${ }^{17}$, Paige M. Bracci ${ }^{18}$, Alexandra Nieters ${ }^{19}$, Roel C. H. Vermeulen ${ }^{20,21}$, Joseph Vijai ${ }^{22}$, Karin E. Smedby ${ }^{23,24}$, Yawei Zhang ${ }^{25}$, Claire M. Vajdic ${ }^{26}$, Wendy Cozen ${ }^{27,28,}$ John J. Spinelli ${ }^{29,30}$, Henrik Hjalgrim ${ }^{31}$, Graham G. Giles ${ }^{11,32}$, Brian K. Link ${ }^{33}$, Jacqueline Clavel ${ }^{8,9}$, Alan A. Arslan ${ }^{34,35,36}$, Mark P. Purdue ${ }^{37}$, Lesley F. Tinker ${ }^{38}$, Demetrius Albanes ${ }^{1}$, Giovanni M. Ferri ${ }^{39}$, Thomas M. Habermann ${ }^{40}$, Hans-Olov Adami ${ }^{41,42}$, Nikolaus Becker ${ }^{43}$, Yolanda Benavente ${ }^{44,45}$, Simonetta Bisanzi ${ }^{46}$, Paolo Boffetta ${ }^{47}$, Paul Brennan ${ }^{48}$, Angela R. Brooks-Wilson ${ }^{49,50}$, Federico Canzian ${ }^{51}$, Lucia Conde ${ }^{52}$, David G. Cox ${ }^{53}$, Karen Curtin ${ }^{54}$, Lenka Foretova ${ }^{55}$, Susan M. Gapstur ${ }^{7}$, Hervé Ghesquières ${ }^{16,56}$, Martha Glenn ${ }^{57}$, Bengt Glimelius ${ }^{58}$, Rebecca D. Jackson ${ }^{59}$, Qing Lan ${ }^{1}$, Mark Liebow ${ }^{40}$, Marc Maynadie ${ }^{60}$, James McKay ${ }^{48}$, Mads Melbye ${ }^{31,61,}$ Lucia Miligi ${ }^{62}$, Roger L. Milne ${ }^{11,32}$, Thierry J. Molina ${ }^{63}$, Lindsay M. Morton', Kari E. North ${ }^{64,65}$, Kenneth Offit ${ }^{22}$, Marina Padoan ${ }^{66}$, Alpa V. Patel ${ }^{7}$, Sara Piro ${ }^{62}$, Vignesh Ravichandran ${ }^{22}$, Elio Riboli ${ }^{67}$, Silvia de Sanjose ${ }^{44,45}$, Richard K. Severson ${ }^{68}$, Melissa C. Southey ${ }^{69}$, Anthony Staines ${ }^{70}$, Carolyn Stewart ${ }^{22}$, Ruth C. Travis ${ }^{71}$, Elisabete Weiderpass ${ }^{72}$, Stephanie Weinstein ${ }^{1}$, Tongzhang Zheng ${ }^{73}$, Stephen J. Chanock ${ }^{1}$, Nilanjan Chatterjee ${ }^{1,74,75}$, Nathaniel Rothman ${ }^{1}$, Brenda M. Birmann ${ }^{76}$, James R. Cerhan ${ }^{13}$ and Sonja I. Berndt ${ }^{\text {* }}$
\end{abstract}

${ }^{1}$ Division of Cancer Epidemiology and Genetics, National Cancer Institute, Bethesda, MD, United States, ${ }^{2}$ Department of Health Sciences, University of York, York, United Kingdom, ${ }^{3}$ Department of Computational Biology, St. Jude Children's Research Hospital, Memphis, TN, United States, ${ }^{4}$ Laboratory of Translational Genomics, Division of Cancer Epidemiology and Genetics, National Cancer Institute, Bethesda, MD, United States, ${ }^{5}$ Department of Health Services, Policy, and Practice, Brown University School of Public Health, Providence, RI, United States, ${ }^{6}$ Center for Gerontology and Healthcare Research, Brown University School of Public Health, Providence, RI, United States, ${ }^{7}$ Epidemiology Research Program, American Cancer Society, Atlanta, GA, United States, ${ }^{8}$ Epidemiology of Childhood and Adolescent Cancers Group, Inserm, Center of Research in Epidemiology and Statistics Sorbonne Paris Cité (CRESS), Paris, France, ${ }^{9}$ Université Paris Descartes, Paris, France, ${ }^{10}$ Registre des hémopathies malignes de la Gironde, Institut Bergonié, Bordeaux, France, ${ }^{11}$ Cancer Epidemiology and Intelligence Division, Cancer Council Victoria, Melbourne, VIC, Australia, ${ }^{12}$ Department of Hematology and Medical Oncology, Emory University School of Medicine, Atlanta, GA, United States, ${ }^{13}$ Department of Health Sciences Research, Mayo Clinic, Rochester, MN, United States, ${ }^{14}$ Department of Hematology, Hospices Civils de Lyon, Lyon, France, ${ }^{15}$ Department of Hematology, Université Lyon-1, Lyon, France, ${ }^{16}$ Equipe Experimental and Clinical Models of Lymphomagenesis, Cancer Research Center of Lyon, Institut National de Santé et de la Recherche Médicale UMR1052 Pierre Benite, Lyon, France, ${ }^{17}$ Division of Hematology and Hematologic Malignancies, Department of Internal Medicine and Huntsman Cancer Institute, University of Utah School of Medicine, Salt Lake City, UT, United States, ${ }^{18}$ Department of Epidemiology and Biostatistics, University of California, San Francisco, San Francisco, CA, United States, ${ }^{19}$ Center for Chronic Immunodeficiency, University Medical Center Freiburg, Freiburg im Breisgau, Germany, ${ }^{20}$ Division of Environmental Epidemiology, Institute for Risk Assessment Sciences, Utrecht University, Utrecht, Netherlands, ${ }^{21}$ Julius Center for Health Sciences and Primary Care, University Medical Center Utrecht, Utrecht, Netherlands, ${ }^{22}$ Clinical Genetics Service, Department of Medicine, Memorial Sloan Kettering Cancer Center, New York, NY, United States, ${ }^{23}$ Department of Medicine, Solna, Karolinska Institutet, Stockholm, Sweden, ${ }^{24}$ Hematology Center, Karolinska University Hospital, Stockholm, Sweden, ${ }^{25}$ Department of Environmental Health Sciences, Yale School of Public Health, New Haven, CT, United States, ${ }^{26}$ Centre for Big Data Research in Health, University of New South Wales, Sydney, NSW, Australia, ${ }^{27}$ Department of Preventive Medicine, USC Keck School of Medicine, University of Southern California, Los Angeles, CA, United States, ${ }^{28}$ Norris Comprehensive Cancer Center, USC Keck School of Medicine, University of Southern California, Los Angeles, CA, United States, ${ }^{29}$ Cancer Control Research, BC Cancer, Vancouver, BC, Canada, ${ }^{30}$ School of Population and Public Health, University of British Columbia, Vancouver, BC, Canada, ${ }^{31}$ Division of Health Surveillance and Research, Department of Epidemiology Research, Statens Serum Institut, Copenhagen, Denmark, ${ }^{32}$ Centre for Epidemiology and Biostatistics, Melbourne School of Population and Global Health, University of Melbourne, Melbourne, VIC, Australia, ${ }^{33}$ Department of Internal Medicine, Carver College of Medicine, The University of lowa, lowa City, IA, United States, ${ }^{34}$ Department of Obstetrics and Gynecology, New York University School of Medicine, New York, NY, United States, ${ }^{35}$ Department of Environmental Medicine, New York University School of Medicine, New York, NY, United States, ${ }^{36}$ Perlmutter Cancer Center, NYU Langone Medical Center, New York, NY, United States, ${ }^{37}$ Ontario Health Study, Toronto, ON, Canada, ${ }^{38}$ Division of Public Health Sciences, Fred Hutchinson Cancer Research Center, Seattle, WA, United States, 


\begin{abstract}
${ }^{39}$ Interdisciplinary Department of Medicine, University of Bari, Bari, Italy, ${ }^{40}$ Division of General Internal Medicine, Mayo Clinic College of Medicine and Science, Rochester, MN, United States, ${ }^{41}$ Department of Medical Epidemiology and Biostatistics, Karolinska Institutet, Stockholm, Sweden, ${ }^{42}$ Department of Epidemiology, Harvard School of Public Health, Boston, MA, United States, ${ }^{43}$ Division of Cancer Epidemiology, German Cancer Research Center, Heidelberg, Germany, ${ }^{44}$ Cancer Epidemiology Research Programme, Catalan Institute of Oncology-IDIBELL, L'Hospitalet de Llobregat, Barcelona, Spain, ${ }^{45}$ CIBER de Epidemiología y Salud Pública, Barcelona, Spain, ${ }^{46}$ Regional Cancer Prevention Laboratory, Oncological Network, Prevention and Research Institute (ISPRO), Florence, Italy, ${ }^{47}$ The Tisch Cancer Institute, Icahn School of Medicine at Mount Sinai, New York, NY, United States, ${ }^{48}$ International Agency for Research on Cancer (IARC), Lyon, France, ${ }^{49}$ Genome Sciences Centre, BC Cancer, Vancouver, BC, Canada, ${ }^{50}$ Department of Biomedical Physiology and Kinesiology, Simon Fraser University, Burnaby, BC, Canada, ${ }^{51}$ Genomic Epidemiology Group, German Cancer Research Center, Heidelberg, Germany, ${ }^{52}$ Bill Lyons Informatics Centre, UCL Cancer Institute, University College London, London, United Kingdom, ${ }^{53}$ INSERM U1052, Cancer Research Center of Lyon, Centre Léon Bérard, Lyon, France, ${ }^{54}$ Department of Internal Medicine, University of Utah School of Medicine, Salt Lake City, UT, United States, ${ }^{55}$ Department of Cancer Epidemiology and Genetics, Masaryk Memorial Cancer Institute and MF MU, Brno, Czechia, ${ }^{56}$ Department of Hematology, Centre Léon Bérard, Lyon, France, ${ }^{57}$ Department of Internal Medicine, Huntsman Cancer Institute, Salt Lake City, UT, United States, ${ }^{58}$ Department of Immunology, Genetics and Pathology, Uppsala University, Uppsala, Sweden, ${ }^{59}$ Division of Endocrinology, Diabetes and Metabolism, The Ohio State University, Columbus, OH, United States, ${ }^{6}$ INSERM U1231, Registre des Hémopathies Malignes de Côte d'Or, University of Burgundy and Dijon University Hospital, Dijon, France, ${ }^{61}$ Department of Medicine, Stanford University School of Medicine, Stanford, CA, United States, ${ }^{62}$ Environmental and Occupational Epidemiology Unit, Oncological Network, Prevention and Research Institute (ISPRO), Florence, Italy, ${ }^{63}$ Department of Pathology, AP-HP, Necker Enfants malades, Université Paris Descartes, Sorbonne Paris Cité, Paris, France, ${ }^{64}$ Department of Epidemiology, University of North Carolina at Chapel Hill, Chapel Hill, NC, United States, ${ }^{65}$ Carolina Center for Genome Sciences, University of North Carolina at Chapel Hill, Chapel Hill, NC, United States, ${ }^{66}$ CPO-Piemonte and Unit of Medical Statistics and Epidemiology, Department Translational Medicine, University of Piemonte Orientale, Novara, Italy, ${ }^{67}$ School of Public Health, Imperial College London, London, United Kingdom, ${ }^{68}$ Department of Family Medicine and Public Health Sciences, Wayne State University, Detroit, MI, United States, ${ }^{69}$ Genetic Epidemiology Laboratory, Department of Pathology, University of Melbourne, Melbourne, VIC, Australia, ${ }^{70}$ School of Nursing and Human Sciences, Dublin City University, Dublin, Ireland, ${ }^{71}$ Cancer Epidemiology Unit, University of Oxford, Oxford, United Kingdom, ${ }^{72}$ International Agency for Research on Cancer, World Health Organization, Lyon, France, ${ }^{73}$ Department of Epidemiology, Brown School of Public Health, Providence, Rl, United States,

${ }^{74}$ Department of Biostatistics, Bloomberg School of Public Health, Johns Hopkins University, Baltimore, MD, United States,

${ }^{75}$ Department of Oncology, School of Medicine, Johns Hopkins University, Baltimore, MD, United States, ${ }^{76}$ Channing Division of Network Medicine, Department of Medicine, Brigham and Women's Hospital and Harvard Medical School, Boston, MA, United States
\end{abstract}

Although the evidence is not consistent, epidemiologic studies have suggested that taller adult height may be associated with an increased risk of some non-Hodgkin lymphoma (NHL) subtypes. Height is largely determined by genetic factors, but how these genetic factors may contribute to $\mathrm{NHL}$ risk is unknown. We investigated the relationship between genetic determinants of height and $\mathrm{NHL}$ risk using data from eight genome-wide association studies (GWAS) comprising 10,629 NHL cases, including 3,857 diffuse large B-cell lymphoma (DLBCL), 2,847 follicular lymphoma (FL), 3,100 chronic lymphocytic leukemia (CLL), and 825 marginal zone lymphoma (MZL) cases, and 9,505 controls of European ancestry. We evaluated genetically predicted height by constructing polygenic risk scores using 833 height-associated SNPs. We used logistic regression to estimate odds ratios $(\mathrm{OR})$ and $95 \%$ confidence intervals $(\mathrm{Cl})$ for association between genetically determined height and the risk of four NHL subtypes in each GWAS and then used fixed-effect meta-analysis to combine subtype results across studies. We found suggestive evidence between taller genetically determined height and increased $\mathrm{CLL}$ risk $(\mathrm{OR}=1.08,95 \% \mathrm{Cl}=1.00-1.17, p=0.049)$, which was slightly stronger among women $(\mathrm{OR}=1.15,95 \% \mathrm{Cl}: 1.01-1.31, p=0.036)$. No significant associations were observed with DLBCL, FL, or MZL. Our findings suggest that there may be some shared genetic factors between CLL and height, but other endogenous or environmental factors may underlie reported epidemiologic height associations with other subtypes.

Keywords: non-Hodgkin lymphoma, height, genetics, chronic lymphocytic leukemia, diffuse large B-cell lymphoma, follicular lymphoma, marginal zone lymphoma, polygenic risk score

\section{INTRODUCTION}

In epidemiologic studies, taller adult height has been associated with an increased risk of several subtypes of non-Hodgkin lymphoma (NHL) (1); however, the results have not been consistent across studies for common subtypes, such as diffuse large B-cell lymphoma (DLBCL), follicular lymphoma (FL), and chronic lymphocytic leukemia/small cell lymphoma (CLL) 
(2-13). Taller height has also been positively associated with other cancers in both epidemiologic and Mendelian randomization studies (14-16). The underlying reason or biological mechanism for these associations is not understood. Adult attained height is not thought to be causally related to cancer, but instead thought to be a marker of other biological influences, including hormonal and growth factors, cellular divisions, and nutrition during formative years, that may contribute to cancer risk over the life course of an individual.

The reported positive associations between height and multiple subtypes of NHL suggest the existence of common biological pathways, possibly mediated by genetic factors. Height is a highly polygenic trait and estimated to be $70-80 \%$ heritable based on twin studies (17-19). Genome-wide association studies (GWAS) have identified hundreds of common variants associated with height to date, which explain approximately $16 \%$ of the variance of height (20), with additional rare variants also contributing to its variance (21). A deeper understanding of how height is associated with NHL risk may provide insight into underlying biological mechanisms leading to lymphoma.

In this study, we used previously established height-related genetic variants to construct polygenic risk scores (PRS) for height in order to examine the associations between geneticallyinferred height and the risk of four common NHL subtypes: DLBCL, FL, CLL, and MZL.

\section{METHODS}

We used data from eight GWAS of NHL subtypes in populations of European ancestry (Supplementary Table 1), the details of which have been reported previous for DLBCL (22), FL (23), CLL (24), and MZL (25). The largest GWAS, the National Cancer Institute (NCI) NHL GWAS, consisted of cases and controls of European ancestry from 22 studies of NHL, including nine prospective cohort studies, eight populationbased case-control studies, and five hospital or clinic-based casecontrol or case-series studies. The other seven GWAS consisted of two population-based case-control studies, one combined clinic- and population-based case-control study, one clinicbased case-control study with additional registry cases, one case series with population-based controls, one randomized clinical trial with population-based controls, and one case-control study enriched for familial cases (Supplementary Table 1). The 29 epidemiologic studies contributing to these GWAS were conducted in the North America, Australia, and Europe. In total, genotype data were available for 3,100 CLL cases, 3,857 DLBCL cases, 2,847 FL cases, and 825 MZL cases, and 9,505 controls. All studies obtained informed consent from participants and were approved by their appropriate Institutional Review Boards.

Genotyping was performed on commercially available Illumina and Affymetrix platforms (Supplementary Table 1). Standard quality control and filtering were applied to each GWAS, and each GWAS was imputed separately. Imputation was performed using IMPUTE2 (26) and the 1,000 Genomes Project version 2, February 2012 release (27) as the reference panel. As reported previously (22-25), quantile-quantile plots of the results showed no substantial evidence of inflation.
Previous studies have reported 848 independent, autosomal SNPs to be associated with height in primarily in European populations $(20,21)$. Wood et al. (20) identified 697 independent SNPs associated with height based on an approximate conditional analysis, taking the linkage disequilibrium among SNPs into account. Marouli et al. (21) identified an additional 113 novel autosomal loci, which were $>1 \mathrm{Mb}$ from the loci reported by Wood et al. and 38 SNPs near established loci that were shown to be independent of previously reported SNPs based on conditional analyses. We constructed weighted polygenic risk scores (PRS) using 833 of these previously reported SNPs, which were available in our datasets with information scores $>0.3$ and $p$-values for Hardy-Weinberg equilibrium $>1 \times 10^{-5}$. To generate the PRS, the height-increasing allelic dosage for each SNP was multiplied by the reported meta-analysis beta coefficient $(20,21)$, as shown below, where $w_{j}$ is the weight or beta coefficient for the $j$ th SNP derived from the literature and $\mathrm{x}_{\mathrm{ij}}$ is the allelic dosage of the jth SNP.

$$
\operatorname{PRS}_{i}=\sum_{j=1}^{k} w_{j} x_{i j}
$$

As previous studies have shown etiologic heterogeneity among NHL subtypes (1), we analyzed each NHL subtype separately. For each GWAS, we performed logistic regression with the PRS, which was normally distributed, modeled as a continuous variable. Models were adjusted for sex, age, and statistically significant principal components for population stratification $(p$ $<0.05)$ at the GWAS level. Since all controls in the UCSF1/NHS GWAS were female, models for this GWAS were adjusted for age and significant principal components of ancestry only. In a sensitivity analysis, we also adjusted for genetically determined body mass index (BMI), using previously established loci. The regression analyses were performed in R using the $\mathrm{glm}$ function.

For each NHL subtype, GWAS-specific risk estimates were pooled using fixed-effect meta-analysis (28). Only one GWAS contributed to MZL, so no meta-analysis was performed for MZL. Between-GWAS heterogeneity was evaluated by the Cochran's Q test and quantified using the $I^{2}$ metric (29). Metaanalyses were conducted using the package metan in Stata v15 (StataCorp, College Station, TX). The UCSF1/NHS GWAS was excluded from the sex-specific meta-analysis of FL because all controls were female. Between-sex heterogeneity was evaluated by performing a fixed-effect meta-analysis to obtain the $p$-value from the Cochran's Q-test and $I^{2}$.

\section{RESULTS}

We found suggestive evidence for an association between taller genetically determined height and risk of CLL $(\mathrm{OR}=1.08$, 95\% CI 1.00-1.17, $p=0.049$ ), with the PRS modeled as a continuous variable (Table 1). Additional adjustment for BMI did not substantially alter the association $(\mathrm{OR}=1.07,95 \%$ CI 0.99-1.16). This association was stronger among females $(\mathrm{OR}=1.15,95 \% \mathrm{CI} 1.01-1.31, p=0.04)$ than among males (OR $=1.06,95 \%$ CI $0.96-1.18, p=0.26)$, but the between-sex heterogeneity was not statistically significant $(p=0.34)$. No 
TABLE 1 | Association between genetically predicted height and the risk of NHL by subtype.

\begin{tabular}{|c|c|c|c|c|c|c|}
\hline & $\begin{array}{c}\text { No. cases/ No. } \\
\text { controls }\end{array}$ & OR & $95 \% \mathrm{Cl}$ & $P$ & $I^{2^{\star}}(\%)$ & $\mathbf{P}_{\text {het }}^{*}$ \\
\hline \multicolumn{7}{|l|}{ CLL } \\
\hline Men + Women & $3100 / 7666$ & 1.08 & $1.00-1.17$ & 0.049 & 0.0 & 0.690 \\
\hline Men & $1794 / 5370$ & 1.06 & $0.96-1.18$ & 0.264 & 0.0 & 0.444 \\
\hline Women & $1306 / 2296$ & 1.15 & $1.01-1.31$ & 0.036 & 0.0 & 0.732 \\
\hline \multicolumn{7}{|l|}{ DLBCL } \\
\hline Men + Women & $3587 / 7666$ & 1.05 & $0.97-1.14$ & 0.209 & 27.2 & 0.249 \\
\hline Men & $1968 / 5260$ & 1.02 & $0.92-1.13$ & 0.731 & 2.1 & 0.382 \\
\hline Women & 1889/2406 & 1.08 & $0.96-1.22$ & 0.205 & 0.0 & 0.539 \\
\hline \multicolumn{7}{|l|}{ FL } \\
\hline Men + Women & $2847 / 8107$ & 1.06 & $0.97-1.16$ & 0.230 & 57.5 & 0.070 \\
\hline Men $^{\dagger}$ & $1276 / 5208$ & 1.03 & $0.91-1.17$ & 0.667 & 0.0 & 0.515 \\
\hline Women $^{\dagger}$ & $1452 / 2550$ & 1.05 & $0.92-1.20$ & 0.470 & 67.2 & 0.048 \\
\hline \multicolumn{7}{|l|}{ MZL } \\
\hline Men + Women & $825 / 6221$ & 1.10 & $0.95-1.28$ & 0.201 & & \\
\hline Men & $334 / 4527$ & 1.19 & $0.96-1.49$ & 0.116 & & \\
\hline Women & 491/1694 & 1.00 & $0.82-1.23$ & 0.967 & & \\
\hline
\end{tabular}

*Heterogeneity among GWAS. Only one GWAS contributed to MZL, so heterogeneity not evaluated for that subtype.

${ }^{\dagger}$ Sex-specific FL analysis excludes UCSF1/NHS study.

significant heterogeneity among studies was observed for CLL among males and females combined $\left(I^{2}=0 \%\right.$, phet $=0.69$, Supplementary Table 2).

No association was observed between genetically predicted height and the risk of DLBCL (OR $=1.05,95 \%$ CI $0.97-1.14$ ), FL $(\mathrm{OR}=1.06,95 \%$ CI $0.97-1.16)$, or MZL $(\mathrm{OR}=1.10,95 \%$ CI 0.95-1.28) (Table 1). Adjustment for BMI did not alter these results (data not shown). For FL, there was some heterogeneity in the GWAS-specific risk estimates $\left(I^{2}=57.5 \%\right.$, phet $=0.07$, Supplementary Table 2), attributable primarily to the inclusion of the SCALE study. Removal of SCALE from the meta-analysis for FL risk slightly strengthened the combined association, but the resulting association did not reach statistical significance (OR $=1.09,95 \%$ CI $0.99-1.20, p=0.08)$. With regards to sex-specific analyses for these three subtypes, no significant associations were seen among men or women, with risk estimates similar to those observed for both sexes combined.

\section{DISCUSSION}

In this first examination of genetically inferred height and NHL risk, we observed a borderline positive association between genetically inferred taller height and the risk of CLL. This finding is consistent with observations from epidemiologic studies examining height and CLL risk, which generally have reported positive or insignificant associations $(2,3,5,11)$. The prospective UK Million Women Study, with 920 CLL cases, found an increased risk of CLL with increasing height (2). Although the Netherlands Cohort Study observed an overall association between taller adult height and risk of NHL overall, it did not observe a significantly elevated risk of CLL with 165
CLL cases (5). A previous pooled analysis within the InterLymph Consortium found an overall positive association between reported height and risk of CLL with a modest magnitude of association (OR per $10-\mathrm{cm}$ increase $=1.10$, 95\% CI $=1.02$ 1.19) (11), which is comparable to the association with a oneunit increase in the PRS from the present study, but without a suggestion of heterogeneity by sex. Collectively, the present and published data provide evidence for a positive association between adult height and risk of CLL, although additional studies are needed to clarify possible sex differences.

No evidence of association was observed between genetically determined height and the risk of DLBCL, FL, nor MZL. Although the findings are not consistent $(3,8)$, some epidemiologic studies have reported positive findings for common NHL subtypes and height $(2,30)$. The UK Million Women Study reported a positive association with height for FL, DLBCL, and NHL overall (2). A large pooled analysis within the InterLymph Consortium found that greater adult height was positively associated FL for males, but not females (12). Consistent with our results, no significant associations were reported between adult height and MZL or DLBCL in the InterLymph pooled analysis $(13,31)$; however, an increased risk of DLBCL was observed for both sexes combined comparing the highest with the lowest quartile of usual adult height in a minimally adjusted model (31). It should be noted that some of the cases and controls included in our analysis were also included in the InterLymph pooled analyses of reported height and NHL risk (32); however, our study included 2,550 cases and 4,330 controls from prospective studies as well, which were not part of the pooled InterLymph analyses (Supplementary Table 1).

Despite the suggestive associations in the literature for selfreported or measured height and FL and DLBCL risk, there are several possible reasons why our study of genetically determined adult height did not identify statistically significant associations. A recent methodologic paper on the use of polygenic risk scores demonstrated that null results are often explained by inadequate sample size (33). Even though our study utilized data from the largest GWAS of NHL to date, it is a possible that our study was underpowered to detect an association for these NHL subtypes. In addition, it is possible that the genetic component of height that is pleiotropically related to risk of these NHL subtypes is not well-captured by the current set of height-associated variants. The PRS for height constructed for the present analyses accounts for $<20 \%$ of the heritable component of adult height $(20,21)$. A PRS incorporating a larger fraction of the heritable component of adult height would provide a more precise measure of the genetic contribution to height and allow for a better assessment of the association with NHL risk. As more of the genetic component of height becomes identified, it may be possible to create a better PRS that accounts for a larger percentage of height variation. Finally, it is also possible that it is the non-genetic component of height, such as childhood nutrition, or a factor correlated with height that is responsible for the reported epidemiologic associations between adult stature and risk of NHL.

Among the noteworthy limitations of our study is the fact that our participants were from populations of European descent, limiting the generalizability of results. However, this restriction 
to Europeans also has the benefit of minimizing bias from population stratification, which is an important concern with studies of height. We did not adjust for multiple testing, because previous studies have shown individual NHL subtypes to have distinct etiologies (1). However, it is possible that the finding we observed with CLL may be a false-positive result, and additional studies are needed to replicate our findings. We were unable to perform a true Mendelian randomization analysis due to lack of data on height from many studies, but we were able to combine multiple GWAS of NHL by meta-analysis to achieve sample sizes of several thousand cases for three of the four subtypes. This approach further enabled a direct assessment of between-study and between-sex heterogeneity of the observed associations. Future studies with even larger sample sizes may be able to evaluate the shared genetic correlation with height using cross-trait LD Score regression.

In conclusion, we present the first polygenic risk score analysis examining the genetic contribution of attained adult height on the risk for the four most common NHL subtypes. Although we did not observe statistically significant associations with DLBCL, FL, or MZL, we observed evidence of a positive association between genetically-inferred height and CLL risk, lending more support to previous observational studies. Additional studies are warranted to explore the association between geneticallyinferred height and risk of other NHL subtypes, particularly those that have been reported to be associated with selfreported or measured height in epidemiologic studies $(34,35)$. Additional insight could be gained from studies that explore the relationship between environmental or endogenous factors that may be correlated with or determinants of height and NHL risk. As more knowledge is gained about the genetic architecture and determinants of height, a more informative PRS can be constructed to represent a larger fraction of the heritable component of adult height and underlying biological pathways. A re-examination of the associations of NHL subtypes with genetically-inferred height may be merited in the future as our understanding of height and its underlying biology grows.

\section{DATA AVAILABILITY STATEMENT}

The datasets generated for this study can be found in the dbGap phs000801.v2.p1 at https://www.ncbi.nlm.nih.gov/projects/gap/ cgi-bin/study.cgi?study_id=phs000801.v2.p1.

\section{ETHICS STATEMENT}

All studies involving human participants were reviewed and approved by their respective Institutional Review Boards. The patients/participants provided their written informed consent to participate in this study.

\section{AUTHOR CONTRIBUTIONS}

AMoo and SIB organized and designed the study. AMoo, EK, LRT, MJM, OP, AMon, NW, ZW, CFS, JRC, and SIB contributed to the design and execution of data analysis. AMoo and SIB wrote the first draft of the manuscript. EK, LRT, AMon, NW, CFS, SLS, RV, CV, YZ, KS, JS, VJ, GG, BL, AA, AN, PMB, NJC, GS, WC, HH, H-OA, DA, NB, YB, SB, PBo, PBr, AB-W, FC, JC, LC, DC, KC, GF, LF, SG, HG, MG, BG, TH, RJ, QL, ML, MMa, JM, MMe, LM, RM, TM, LMM, KN, KO, MPu, AP, VR, ER, SS, RS, MS, AS, LFT, RT, EW, SW, TZ, NR, BB, JRC, and SIB conducted the epidemiological studies involved this study. All authors contributed to the writing of the manuscript.

\section{FUNDING}

This work was supported by the Intramural Research Program of the Division of Cancer Epidemiology and Genetics, National Cancer Institute. Support for individual studies:

ATBC-The ATBC Study was supported by the Intramural Research Program of the U.S. National Cancer Institute, National Institutes of Health, and by U.S. Public Health Service contract HHSN261201500005C.

BC-Canadian Institutes for Health Research (CIHR); Canadian Cancer Society; Michael Smith Foundation for Health Research.

CPS-II-The Cancer Prevention Study-II (CPS-II) Nutrition Cohort was supported by the American Cancer Society. Genotyping for all CPS-II samples were supported by the Intramural Research Program of the National Institutes of Health, NCI, Division of Cancer Epidemiology and Genetics. The authors would also like to acknowledge the contribution to this study from central cancer registries supported through the Centers for Disease Control and Prevention National Program of Cancer Registries, and cancer registries supported by the National Cancer Institute Surveillance Epidemiology and End Results program.

\section{ELCCS-Bloodwise, United Kingdom.}

ENGELA-Association pour la Recherche contre le Cancer (ARC), Institut National du Cancer (INCa), Fondation de France, Fondation contre la Leucémie, Agence nationale de sécurité sanitaire de l'alimentation, de l'environnement et du travail (ANSES).

EPIC-Coordinated Action (Contract \#006438, SP23-CT-2005006438); HuGeF (Human Genetics Foundation), Torino, Italy; Cancer Research UK.

EpiLymph-European Commission (Grant references QLK4CT-2000-00422 and FOOD-CT-2006-023103); the Spanish Ministry of Health (Grant references CIBERESP, PI11/01810, PI14/01219, RCESP C03/09, RTICESP C03/10 and RTIC RD06/0020/0095), the Marató de TV3 Foundation (Grant reference 051210), the Agència de Gestiód'AjutsUniversitarisi de Recerca-Generalitat de Catalunya (Grant reference 2017SGR1085) who had no role in the data collection, analysis or interpretation of the results; the NIH (contract NO1-CO12400); the Compagnia di San Paolo-Programma Oncologia; the Federal Office for Radiation Protection grants StSch4261 and StSch4420, the José Carreras Leukemia Foundation grant DJCLS-R12/23, the German Federal Ministry for Education 
and Research (BMBF-01-EO-1303); the Health Research Board, Ireland and Cancer Research Ireland; Czech Republic supported by MH CZ-DRO (MMCI, 00209805) and MEYS-NPS ILO1413; Fondation de France and Association de Recherche Contre le Cancer.

GEC/Mayo GWAS-National Institutes of Health (CA118444, CA148690, CA92153). Intramural Research Program of the NIH, National Cancer Institute. Veterans Affairs Research Service. Data collection for Duke University was supported by a Leukemia \& Lymphoma Society Career Development Award, the Bernstein Family Fund for Leukemia and Lymphoma Research, and the National Institutes of Health (K08CA134919), National Center for Advancing Translational Science (UL1 TR000135).

HPFS (Walter C. Willet)-The HPFS was supported in part by National Institutes of Health grants CA167552, CA149445, and CA098122. We would like to thank the participants and staff of the Health Professionals Follow-up Study for their valuable contributions as well as the following state cancer registries for their help: AL, AZ, AR, CA, CO, CT, DE, FL, GA, ID, IL, IN, IA, KY, LA, ME, MD, MA, MI, NE, NH, NJ, NY, NC, ND, OH, OK, OR, PA, RI, SC, TN, TX, VA, WA, WY. The authors assume full responsibility for analyses and interpretation of these data.

Iowa-Mayo SPORE-NCI Specialized Programs of Research Excellence (SPORE) in Human Cancer (P50 CA97274); National Cancer Institute (P30 CA086862, P30 CA15083); Henry J. Predolin Foundation.

Italian GxE-Italian Association for Cancer Research (AIRC, Investigator Grant 11855) (PC); Fondazione Banco di Sardegna 2010-2012, and Regione Autonoma della Sardegna (LR7 CRP59812/2012) (MGE).

Mayo Clinic Case-Control-National Institutes of Health (R01 CA92153); National Cancer Institute (P30 CA015083).

MCCS-The Melbourne Collaborative Cohort Study recruitment was funded by VicHealth and Cancer Council Victoria. The MCCS was further supported by Australian NHMRC grants 209057, 251553, and 504711 and by infrastructure provided by Cancer Council Victoria. Cases and their vital status were ascertained through the Victorian Cancer Registry and the Australian Institute of Health and Welfare, including the National Death Index and the Australian Cancer Database.

MSKCC-Geoffrey Beene Cancer Research Grant, Lymphoma Foundation (LF5541); Barbara K. Lipman Lymphoma Research Fund (74419); Robert and Kate Niehaus Clinical Cancer Genetics Research Initiative (57470).

NCI-SEER-Intramural Research Program of the National Cancer Institute, National Institutes of Health, and Public Health Service (N01-PC-65064, N01-PC-67008, N01-PC-67009, N01PC-67010, N02-PC-71105).

NHS (Meir J. Stampfer)-The NHS was supported in part by National Institutes of Health grants CA186107, CA87969, CA49449, CA149445, CA098122, and CA134958. We would like to thank the participants and staff of the Nurses' Health Study for their valuable contributions as well as the following state cancer registries for their help: AL, AZ, AR, CA, CO, CT, DE, FL, GA, ID, IL, IN, IA, KY, LA, ME, MD, MA, MI, NE, NH, NJ, NY, NC, ND, OH, OK, OR, PA, RI, SC, TN, TX, VA, WA, WY. The authors assume full responsibility for analyses and interpretation of these data.

NSW-NSW was supported by grants from the Australian National Health and Medical Research Council (ID990920), the Cancer Council NSW, and the University of Sydney Faculty of Medicine.

NYU-WHS-National Cancer Institute (R01 CA098661, P30 CA016087); National Institute of Environmental Health Sciences (ES000260).

PLCO-This research was supported by the Intramural Research Program of the National Cancer Institute and by contracts from the Division of Cancer Prevention, National Cancer Institute, NIH, DHHS.

SCALE-Swedish Cancer Society (2009/659). Stockholm County Council (20110209) and the Strategic Research Program in Epidemiology at Karolinska Institutet. Swedish Cancer Society grant (02 6661). National Institutes of Health (5R01 CA6966902); Plan Denmark.

UCSF2-The UCSF studies were supported by the NCI, National Institutes of Health, CA1046282 and CA154643. The collection of cancer incidence data used in this study was supported by the California Department of Health Services as part of the statewide cancer reporting program mandated by California Health and Safety Code section 103885; the National Cancer Institute's Surveillance, Epidemiology, and End Results Program under contract HHSN261201000140C awarded to the Cancer Prevention Institute of California, contract HHSN261201000035C awarded to the University of Southern California, and contract HHSN261201000034C awarded to the Public Health Institute; and the Centers for Disease Control and Prevention's National Program of Cancer Registries, under agreement \#1U58 DP000807-01 awarded to the Public Health Institute. The ideas and opinions expressed herein are those of the authors, and endorsement by the State of California, the California Department of Health Services, the National Cancer Institute, or the Centers for Disease Control and Prevention or their contractors and subcontractors is not intended nor should be inferred.

UTAH/Sheffield-National Institutes of Health CA134674. Partial support for data collection at the Utah site was made possible by the Utah Population Database (UPDB) and the Utah Cancer Registry (UCR). Partial support for all datasets within the UPDB is provided by the Huntsman Cancer Institute (HCI) and the HCI Cancer Center Support grant, P30 CA42014. The UCR was supported in part by NIH contract HHSN261201000026C from the National Cancer Institute SEER Program with additional support from the Utah State Department of Health and the University of Utah. Partial support for data collection in Sheffield, UK was made possible by funds from Yorkshire Cancer 
Research and the Sheffield Experimental Cancer Medicine Centre. We thank the NCRI Haemato-oncology Clinical Studies Group, colleagues in the North Trent Cancer Network the North Trent Haemato-oncology Database.

WHI-WHI investigators are: Program Office-(National Heart, Lung, and Blood Institute, Bethesda, Maryland) Jacques Rossouw, Shari Ludlam, Dale Burwen, Joan McGowan, Leslie Ford, and Nancy Geller; Clinical Coordinating Center-(Fred Hutchinson Cancer Research Center, Seattle, WA) Garnet Anderson, Ross Prentice, Andrea LaCroix, and Charles Kooperberg; Investigators and Academic Centers-(Brigham and Women's Hospital, Harvard Medical School, Boston, MA) JoAnn E. Manson; (MedStar Health Research Institute/Howard University, Washington, DC) Barbara V. Howard; (Stanford Prevention Research Center, Stanford, CA) Marcia L. Stefanick; (The Ohio State University, Columbus, $\mathrm{OH}$ ) Rebecca Jackson; (University of Arizona, Tucson/Phoenix, AZ) Cynthia A. Thomson; (University at Buffalo, Buffalo, NY) Jean WactawskiWende; (University of Florida, Gainesville/Jacksonville, FL) Marian Limacher; (University of Iowa, Iowa City/Davenport, IA) Robert Wallace; (University of Pittsburgh, Pittsburgh, PA) Lewis Kuller; (Wake Forest University School of Medicine, Winston-Salem, NC) Sally Shumaker; Women's Health Initiative Memory Study-(Wake Forest University School of Medicine, Winston-Salem, NC) Sally Shumaker. The WHI

\section{REFERENCES}

1. Morton LM, Slager SL, Cerhan JR, Wang SS, Vajdic CM, Skibola CF, et al. Etiologic heterogeneity among non-Hodgkin lymphoma subtypes: the InterLymph Non-Hodgkin Lymphoma Subtypes Project. J Natl Cancer Inst Monogr. (2014) 2014:130-44. doi: 10.1093/jncimonographs/lgu013

2. Murphy F, Kroll ME, Pirie K, Reeves G, Green J, Beral V. Body size in relation to incidence of subtypes of haematological malignancy in the prospective Million Women Study. Br J Cancer. (2013) 108:2390-8. doi: 10.1038/bjc.2013.159

3. Patel AV, Diver WR, Teras LR, Birmann BM, Gapstur SM. Body mass index, height and risk of lymphoid neoplasms in a large United States cohort. Leuk Lymphoma. (2013) 54:1221-7. doi: 10.3109/10428194.2012.742523

4. Kabat GC, Kim MY, Jean Wactawski W, Bea JW, Edlefsen KL, AdamsCampbell LL, et al. Anthropometric factors, physical activity, and risk of nonHodgkin's lymphoma in the Women's Health Initiative. Cancer Epidemiol. (2012) 36:52-9. doi: 10.1016/j.canep.2011.05.014

5. Pylypchuk RD, Schouten LJ, Goldbohm RA, Schouten HC, van den Brandt PA. Body mass index, height, and risk of lymphatic malignancies: a prospective cohort study. Am J Epidemiol. (2009) 170:297-307. doi: 10.1093/aje/ kwp123

6. Britton JA, Khan AE, Rohrmann S, Becker N, Linseisen J, Nieters A, et al. Anthropometric characteristics and non-Hodgkin's lymphoma and multiple myeloma risk in the European Prospective Investigation into Cancer and Nutrition (EPIC). Haematologica. (2008) 93:1666-77. doi: 10.3324/haematol.13078

7. Lim U, Morton LM, Subar AF, Baris D, Stolzenberg-Solomon R, Leitzmann $\mathrm{M}$, et al. Alcohol, smoking, and body size in relation to incident Hodgkin's and non-Hodgkin's lymphoma risk. Am J Epidemiol. (2007) 166:697-708. doi: 10.1093/aje/kwm122

8. Cerhan JR, Janney CA, Vachon CM, Habermann TM, Kay NE, Potter JD, et al. Anthropometric characteristics, physical activity, and risk of non-Hodgkin's lymphoma subtypes and B-cell chronic lymphocytic leukemia: a prospective study. Am J Epidemiol. (2002) 156:527-35. doi: 10.1093/aje/kwf082 program was funded by the National Heart, Lung, and Blood Institute, National Institutes of Health, U.S. Department of Health and Human Services through contracts HHS N268201600046C, HHSN268201600001C, HHSN26820160 0002C, HHSN268201600003C, HHSN268201600004C, and HHSN271201600004C.

YALE-National Cancer Institute (CA62006); National Cancer Institute (CA165923).

\section{ACKNOWLEDGMENTS}

The authors thank Bill Wheeler, I.M.S., for assistance in performing some of the statistical analysis presented. Where authors are identified as personnel of the International Agency for Research on Cancer/World Health Organization, the authors alone are responsible for the views expressed in this article and they do not necessarily represent the decisions, policy or views of the International Agency for Research on Cancer/World Health Organization.

\section{SUPPLEMENTARY MATERIAL}

The Supplementary Material for this article can be found online at: https://www.frontiersin.org/articles/10.3389/fonc. 2019.01539/full\#supplementary-material

9. Troy JD, Hartge P, Weissfeld JL, Oken MM, Colditz GA, Mechanic LE, et al. Associations between anthropometry, cigarette smoking, alcohol consumption, and non-Hodgkin lymphoma in the Prostate, Lung, Colorectal, and Ovarian Cancer Screening Trial. Am J Epidemiol. (2010) 171:1270-81. doi: 10.1093/aje/kwq085

10. Leiba M, Leiba A, Keinan-Boker L, Avigdor A, Derazne E, Levine H, et al. Adolescent weight and height are predictors of specific nonHodgkin lymphoma subtypes among a cohort of 2,352,988 individuals aged 16 to 19 years. Cancer. (2016) 122:1068-77. doi: 10.1002/cncr. 29792

11. Slager SL, Benavente Y, Blair A, Vermeulen R, Cerhan JR, Costantini AS, et al. Medical history, lifestyle, family history, and occupational risk factors for chronic lymphocytic leukemia/small lymphocytic lymphoma: the InterLymph Non-Hodgkin Lymphoma Subtypes Project. J Natl Cancer Inst Monogr. (2014) 2014:41-51. doi: 10.1093/jncimonographs/lgu001

12. Linet MS, Vajdic CM, Morton LM, de Roos AJ, Skibola CF, Boffetta $\mathrm{P}$, et al. Medical history, lifestyle, family history, and occupational risk factors for follicular lymphoma: the InterLymph Non-Hodgkin Lymphoma Subtypes Project. J Natl Cancer Inst Monogr. (2014) 2014:26-40. doi: 10.1093/jncimonographs/lgu006

13. Bracci PM, Benavente Y, Turner JJ, Paltiel O, Slager SL, Vajdic CM, et al. Medical history, lifestyle, family history, and occupational risk factors for marginal zone lymphoma: the InterLymph Non-Hodgkin Lymphoma Subtypes Project. J Natl Cancer Inst Monogr. (2014) 2014:52-65. doi: 10.1093/jncimonographs/lgu011

14. Thrift AP, Gong J, Peters U, Chang-Claude J, Rudolph A, Slattery ML, et al. Mendelian randomization study of height and risk of colorectal cancer. Int J Epidemiol. (2015) 44:662-72. doi: 10.1093/ije/dyv082

15. Zhang B, Shu XO, Delahanty RJ, Zeng C, Michailidou K, Bolla MK, et al. Height and breast cancer risk: evidence from prospective studies and mendelian randomization. J Natl Cancer Inst. (2015) 107:djv219. doi: 10.1093/jnci/djv219

16. Khankari NK, Shu XO, Wen W, Kraft P, Lindstrom S, Peters U, et al. Association between adult height and risk of colorectal, lung, 
and prostate cancer: results from meta-analyses of prospective studies and Mendelian randomization analyses. PLoS Med. (2016) 13:e1002118. doi: 10.1371/journal.pmed.1002118

17. Silventoinen K, Sammalisto S, Perola M, Boomsma DI, Cornes BK, Davis C, et al. Heritability of adult body height: a comparative study of twin cohorts in eight contries. Twin Res. (2003) 6:399-408. doi: 10.1375/136905203770326402

18. Visscher PM, Medland SE, Ferreira MA, Morley KI, Zhu G, Cornes $\mathrm{BK}$, et al. Assumption-free estimation of heritability from genome-wide identity-by-descent sharing between full siblings. PLoS Genet. (2006) 2:e41. doi: 10.1371/journal.pgen.0020041

19. Jelenkovic A, Sund R, Hur YM, Yokoyama Y, Hjelmborg JV, Moller S, et al. Genetic and environmental influences on height from infancy to early adulthood: an individual-based pooled analysis of 45 twin cohorts. Sci Rep. (2016) 6:28496. doi: 10.1038/srep28496

20. Wood AR, Esko T, Yang J, Vedantam S, Pers TH, Gustafsson S, et al. Defining the role of common variation in the genomic and biological architecture of adult human height. Nat Genet. (2014) 46:1173-86. doi: 10.1038/ng.3097

21. Marouli E, Graff M, Medina-Gomez C, Lo KS, Wood AR, Kjaer TR, et al. Rare and low-frequency coding variants alter human adult height. Nature. (2017) 542:186-90. doi: 10.1038/nature21039

22. Cerhan JR, Berndt SI, Vijai J, Ghesquieres H, McKay J, Wang SS, et al. Genome-wide association study identifies multiple susceptibility loci for diffuse large B cell lymphoma. Nat Genet. (2014) 46:1233-8. doi: 10.1158/1538-7445.AM2014-LB-272

23. Skibola CF, Berndt SI, Vijai J, Conde L, Wang Z, Yeager M, et al. Genome-wide association study identifies five susceptibility loci for follicular lymphoma outside the HLA region. Am J Hum Genet. (2014) 95:462-71. doi: 10.1016/j.ajhg.2014.09.004

24. Berndt SI, Skibola CF, Joseph V, Camp NJ, Nieters A, Wang Z, et al. Genome-wide association study identifies multiple risk loci for chronic lymphocytic leukemia. Nat Genet. (2013) 45:868-76. doi: 10.1158/1538-7445.AM2013-LB-23

25. Vijai J, Wang Z, Berndt SI, Skibola CF, Slager SL, de Sanjose S, et al. A genomewide association study of marginal zone lymphoma shows association to the HLA region. Nat Commun. (2015) 6:5751. doi: 10.1038/ncomms6751

26. Howie BN, Donnelly P, Marchini J. A flexible and accurate genotype imputation method for the next generation of genome-wide association studies. PLoS Genet. (2009) 5:e1000529. doi: 10.1371/journal.pgen.10 00529

27. Genomes Project C, Abecasis GR, Altshuler D, Auton A, Brooks LD, Durbin RM, et al. A map of human genome variation from population-scale sequencing. Nature. (2010) 467:1061-73. doi: 10.1038/nature09534

28. Panagiotou OA, Willer CJ, Hirschhorn JN, Ioannidis JP. The power of metaanalysis in genome-wide association studies. Annu Rev Genomics Hum Genet. (2013) 14:441-65. doi: 10.1146/annurev-genom-091212-153520

29. Higgins JP, Thompson SG. Quantifying heterogeneity in a meta-analysis. Stat Med. (2002) 21:1539-58. doi: 10.1002/sim.1186

30. Cerhan JR, Bernstein L, Severson RK, Davis S, Colt JS, Blair A, et al. Anthropometrics, physical activity, related medical conditions, and the risk of non-hodgkin lymphoma. Cancer Causes Control. (2005) 16:1203-14. doi: 10.1007/s10552-005-0358-7

31. Cerhan JR, Kricker A, Paltiel O, Flowers CR, Wang SS, Monnereau A, et al. Medical history, lifestyle, family history, and occupational risk factors for diffuse large B-cell lymphoma: the InterLymph Non-Hodgkin Lymphoma Subtypes Project. J Natl Cancer Inst Monogr. (2014) 2014:15-25. doi: 10.1093/jncimonographs/lgu010

32. Morton LM, Sampson JN, Cerhan JR, Turner JJ, Vajdic CM, Wang SS, et al. Rationale and design of the international lymphoma epidemiology consortium (InterLymph) non-hodgkin lymphoma subtypes project. J Nat Cancer Inst Monogr. (2014) 2014:1-14. doi: 10.1093/jncimonographs/lgu005

33. Dudbridge F. Power and predictive accuracy of polygenic risk scores. PLoS Genet. (2013) 9:e1003348. doi: 10.1371/journal.pgen.1003348

34. Mbulaiteye SM, Morton LM, Sampson JN, Chang ET, Costas L, de Sanjose S, et al. Medical history, lifestyle, family history, and occupational risk factors for sporadic Burkitt lymphoma/leukemia: the Interlymph Non-Hodgkin Lymphoma Subtypes Project. J Natl Cancer Inst Monogr. (2014) 2014:106-14. doi: 10.1093/jncimonographs/lgu003

35. Monnereau A, Slager SL, Hughes AM, Smith A, Glimelius B, Habermann TM, et al. Medical history, lifestyle, and occupational risk factors for hairy cell leukemia: the InterLymph Non-Hodgkin Lymphoma Subtypes Project. J Natl Cancer Inst Monogr. (2014) 2014:115-24. doi: 10.1093/jncimonographs/lgu004

Conflict of Interest: The authors declare that the research was conducted in the absence of any commercial or financial relationships that could be construed as a potential conflict of interest.

Citation: Moore A, Kane E, Wang Z, Panagiotou OA, Teras LR, Monnereau A, Wong Doo N, Machiela MJ, Skibola CF, Slager SL, Salles G, Camp NJ, Bracci PM, Nieters A, Vermeulen RCH, Vijai J, Smedby KE, Zhang Y, Vajdic CM, Cozen W, Spinelli JJ, Hjalgrim H, Giles GG, Link BK, Clavel J, Arslan AA, Purdue MP, Tinker LF, Albanes D, Ferri GM, Habermann TM, Adami H-O, Becker $N$, Benavente Y, Bisanzi S, Boffetta P, Brennan P, Brooks-Wilson AR, Canzian F, Conde L, Cox DG, Curtin K, Foretova L, Gapstur SM, Ghesquières H, Glenn M, Glimelius B, Jackson RD, Lan Q, Liebow M, Maynadie M, McKay J, Melbye M, Miligi L, Milne RL, Molina TJ, Morton LM, North KE, Offit K, Padoan M, Patel AV, Piro S, Ravichandran V, Riboli E, de Sanjose S, Severson RK, Southey MC, Staines A, Stewart C, Travis RC, Weiderpass E, Weinstein S, Zheng T, Chanock SJ, Chatterjee N, Rothman N, Birmann BM, Cerhan JR and Berndt SI (2020) Genetically Determined Height and Risk of Non-hodgkin Lymphoma. Front. Oncol. 9:1539. doi: 10.3389/fonc.2019.01539

Copyright (c) 2020 Moore, Kane, Wang, Panagiotou, Teras, Monnereau, Wong Doo, Machiela, Skibola, Slager, Salles, Camp, Bracci, Nieters, Vermeulen, Vijai, Smedby, Zhang, Vajdic, Cozen, Spinelli, Hjalgrim, Giles, Link, Clavel, Arslan, Purdue, Tinker, Albanes, Ferri, Habermann, Adami, Becker, Benavente, Bisanzi, Boffetta, Brennan, Brooks-Wilson, Canzian, Conde, Cox, Curtin, Foretova, Gapstur, Ghesquières, Glenn, Glimelius, Jackson, Lan, Liebow, Maynadie, McKay, Melbye, Miligi, Milne, Molina, Morton, North, Offit, Padoan, Patel, Piro, Ravichandran, Riboli, de Sanjose, Severson, Southey, Staines, Stewart, Travis, Weiderpass, Weinstein, Zheng, Chanock, Chatterjee, Rothman, Birmann, Cerhan and Berndt. This is an open-access article distributed under the terms of the Creative Commons Attribution License (CC BY). The use, distribution or reproduction in other forums is permitted, provided the original author(s) and the copyright owner(s) are credited and that the original publication in this journal is cited, in accordance with accepted academic practice. No use, distribution or reproduction is permitted which does not comply with these terms. 